\title{
TETANUS TOXIN: CONVULSANT ACTION ON MOUSE SPINAL CORD NEURONS IN CULTURE'
}

\author{
GREGORY K. BERGEY, ${ }^{*, 2}$ ROBERT L. MACDONALD,${ }^{*, 3}$ WILLIAM H. HABIG, $\ddagger$ M. CAROLYN \\ HARDEGREE, $\$$ AND PHILLIP G. NELSON*
}

${ }^{*}$ Laboratory of Developmental Neurobiology, National Institute of Child Health and Human Development, National Institutes of Health, Bethesda, Maryland 20205 and $\ddagger$ Bacterial Toxins Branch, Bureau of Biologics, Food and Drug Administration, Bethesda, Maryland 20205

Received June 22, 1982; Revised May 23, 1983; Accepted May 25, 1983

\begin{abstract}
The effects of direct application of tetanus toxin on fetal mouse spinal cord neurons in culture are described. Tetanus toxin produces increased excitation characterized by paroxysmal depolarizing events (PDE). In contrast to the abrupt onset of convulsant action produced by the postsynaptic glycine antagonist strychnine, the convulsant action of tetanus occurs after a dose-dependent latent period. The onset of the convulsant action of tetanus toxin is paralleled by a reduction in observed spontaneous inhibitory synaptic potentials. Excitatory synaptic events can be identified as components of some tetanus-PDE. The toxin does not alter postsynaptic responses to the inhibitory amino acids glycine and $\gamma$-aminobutyric acid. The latency and convulsant action of tetanus toxin are consistent with an irreversible presynaptic membrane interaction that reduces inhibitory transmission, a mechanism of action distinct from those of convulsants that antagonize inhibitory transmitters at the postsynaptic membrane.
\end{abstract}

Tetanus toxin, the exquisitely potent product of the Clostridium tetani bacterium, acts to produce disinhibition of the mammalian central nervous system (CNS), particularly in the spinal cord. The clinical presentation of tetanus is one of violent muscle spasms characterized by restricted (local tetanus) or generalized simultaneous agonist-antagonist contractions of the affected muscle groups (Habermann, 1978). Excluded by the blood-brain barrier (Habermann and Dimpfel, 1973), tetanus toxin is taken up by axon terminals at peripheral neuromuscular junctions and reaches the motoneurons of the spinal cord and brainstem by retrograde transport (Erdmann et al., 1975; Price et al., 1975, 1978). Direct spinal cord injections of tetanus toxin result in the reduction of spinal inhibition, including Ia and recurrent Renshaw inhibition (Brooks et al., 1957; Curtis, 1959; Curtis et al., 1973).

The observation that tetanus toxin reduces spinal inhibition while having no demonstrable effect on postsynaptic responses to iontophoretically applied $\gamma$-ami-

\footnotetext{
${ }^{1}$ We wish to thank Sandra Fitzgerald for preparation and maintenance of the cell cultures used in these experiments and Maxine Schaefer for typing the manuscript.

${ }^{2}$ Present address: Departments of Neurology and Physiology, University of Maryland School of Medicine, Baltimore, MD 21201.

${ }^{3}$ Present address: Department of Neurology, University of Michigan School of Medicine, Ann Arbor, MI 48109.
}

nobutyric acid (GABA) or glycine (Curtis et al., 1973) suggests a presynaptic site of action for the toxin. Subsequent reports of central trans-synaptic migration of peripherally applied toxin (Schwab and Thoenen, 1976; Schwab et al., 1979) and presynaptic localization of centrally injected toxin (Price et al., 1977; Price and Griffin, 1981) provide further indirect support for a presynaptic site of action. Following injection of ${ }^{125}$ I-toxin into muscle, intracisternal administration of antitoxin in rats can prevent clinical tetanus despite demonstrable transport of ${ }^{125}$ I-tetanus toxin to motoneurons (Erdmann et al., 1981). In CNS synaptosomal preparations, tetanus toxin has been demonstrated to inhibit potassium-stimulated release of selected neurotransmitters (Bigalke et al., 1981b).

To investigate further the actions of tetanus toxin, we have utilized dissociated fetal mouse spinal cord neurons in culture. The physiologic and morphologic parameters of these neurons in culture have been characterized (Ransom et al., 1977a, b, c; Barker and Ransom, 1978; Macdonald and Barker, 1981; Nelson et al., 1981); abundant excitatory and inhibitory synapses are present. This preparation allows direct access of toxin to neuronal membranes and synaptic structures without requiring axonal transport from the periphery or diffusion through dense neuropil. An additional benefit of this system is the ability to observe the effects of concentrations of 
tetanus toxin over time intervals (potentially days to weeks) that are not possible in intact animals.

We report here the action of tetanus toxin on mammalian spinal cord neurons in cell culture. Tetanus toxin produced prominent paroxysmal depolarizing events (PDE) with associated triggered action potentials. Characteristics of these depolarizing events are presented with supporting evidence that synaptic transmission is important in the central spinal action of tetanus toxin. The action of tetanus toxin is compared to that of strychnine, a known postsynaptic glycine antagonist (Curtis et al., 1971) capable of producing excitation of neurons in cell culture as well as a clinical picture with similarities to that of generalized tetanus (Arena, 1974). A preliminary abstract of some of these observations has been published (Macdonald et al., 1979).

\section{Materials and Methods}

Culture technique. Spinal cords were removed from $12^{1 / 2}$ - to 14-day-old fetal mice, trypsin dissociated, and plated in $35-\mathrm{mm}$ collagen-coated plastic tissue culture dishes as described in detail previously (Ransom et al., 1977c). The culture medium was Eagle's minimal essential medium (MEM) supplemented with glucose (final concentration, $30 \mathrm{mM}$ ) and bicarbonate (final concentration, $44 \mathrm{mM}$ ) to yield a buffered solution ( $\mathrm{pH} 7.2$ to 7.4) when incubated in a $10 \%$ carbon dioxide atmosphere at $35^{\circ} \mathrm{C}$. No antibiotics were used. During the first 7 days of culture, the medium contained $10 \%$ horse serum and $10 \%$ fetal calf serum (FCS). After 6 days the cultures were treated for $24 \mathrm{hr}$ with the antimetabolite 5 -fluoro2 -deoxyuridine (concentration, $54 \mu \mathrm{M}$ with $140 \mu \mathrm{M}$ uridine) to suppress growth of non-neuronal elements and yield neurons free of glial investitures (Ransom et al., 1977b). After the initial 7 days the cultures were maintained in MEM with $10 \%$ horse serum only. Medium was changed (subtotal changes) twice weekly. Cultures at 4 weeks had many neurons with abundant processes. Both dorsal root ganglion (DRG) neurons and spinal cord neurons were present in these cultures.

Because of the high level of tetanus antitoxin present in horse serum (about 10 units (U)/ml in the serum lots used here, as determined by bioassay), attempts were made to eliminate horse serum from the culture medium. Cultures grown in MEM with only FCS (range 1 to $20 \%$ ) had markedly reduced neuronal numbers and viability. Therefore, horse serum was used in the initial weeks of culture. Prior to adding tetanus toxin, cultures were washed three times in MEM with 1\% FCS. Experiments were performed in this medium or, in a few instances, in MEM alone. Assay of the medium after washing revealed no detectable tetanus antitoxin (i.e., less than $0.001 \mathrm{U}$ ) by bioassay. Established spinal cord cultures (over 4 weeks of age) could be maintained in MEM or MEM with $1 \%$ FCS for days without observed detrimental effects.

Spinal cord cultures for fluorescent studies were grown for 4 to 5 weeks on collagen-coated $12-\mathrm{mm}$ glass coverslips under the culture conditions described above.

Tetanus toxin. Homogeneous tetanus toxin $\left(M_{r}\right.$ $\sim$ 150,000; Bizzini et al., 1973) was prepared and purified as described previously (Ledley et al., 1977). Final con- centrations of stock toxin were 2.2 to $5.0 \mathrm{mg} / \mathrm{ml}$ in $0.1 \mathrm{M}$ sodium phosphate buffer. The toxin had 1 to $2 \times 10^{7}$ mouse lethal doses (MLD)/mg of toxin; one MLD is the least amount of toxin which kills a 15- to 18-gm mouse within 4 days after subcutaneous injection into the inguinal fold region. Stock solutions of toxin were stored frozen $\left(-40^{\circ} \mathrm{C}\right)$ until use; no loss of toxicity was detectable with these storage methods. Dilutions of toxin were made in MEM or MEM with 1\% FCS (to minimize nonspecific adherence of toxin). Periodic bioassay of 2fold dilutions of final toxin solutions were performed to confirm calculated toxicity of toxin dilutions.

Equine tetanus antitoxin (TAT) was purchased from Sclavo, Inc. (Wayne, NJ) and was dialyzed against saline to remove preservatives, yielding a stock solution of approximately $200 \mathrm{U} / \mathrm{ml}$ based on the labeled value.

All laboratory personnel had recent tetanus toxoid booster immunizations, and those using toxin preparations had protective antibody titers $(0.01 \mathrm{U} / \mathrm{ml}$ or greater) as determined by a toxin neutralization test (Barile et al., 1970).

Rhodamine-labeled tetanus toxin was prepared by the method of Pastan et al. (1977) with tetramethylrhodamine isothiocyanate (Baltimore Biologicals, Baltimore, MD), yielding a final concentration of toxin of $10^{-4} \mathrm{gm} /$ $\mathrm{ml}$ with retention of bioactivity.

Culture coverslips were washed three times in $1 \%$ FCS/MEM or phosphate-buffered saline with $1 \%$ bovine serum albumin. Rhodamine toxin was added for 10 to 60 min at concentrations of $10^{-4}$ to $10^{-6} \mathrm{gm} / \mathrm{ml}$ at $6^{\circ}$ or $35^{\circ} \mathrm{C}$. Cultures were then washed in $1 \mathrm{M}$ phosphate buffer, fixed in $1 \%$ formaldehyde for $15 \mathrm{~min}$, and observed for fluorescence using epi-illumination on a Zeiss photomicroscope with a BP 510-550 excitation filter and a Kodak 23A barrier filter.

Physiology. Spinal cord cultures used for intracellular recordings were allowed to develop for 4 to 10 weeks to yield spinal cord neurons of sufficient size to be routinely penetrated with intracellular microelectrodes. Cultures were washed as described above. Culture plates were placed on the temperature $\left(33\right.$ to $\left.34^{\circ} \mathrm{C}\right)$ - and $\mathrm{pH}$-controlled $\left(10 \% \quad \mathrm{CO}_{2}\right.$ gassed) stage of an inverted phase microscope. Intracellular recordings were made from penetrations under direct vision using fiber-filled glass micropipettes ( 30 to 50 megohms) filled with $4 \mathrm{M}$ potassium acetate (KAc). A conventional bridge circuit, dual trace oscilloscope and a variable speed continuous multichannel penwriter were used for recordings of neuronal activity and responses to iontophoretically or pressureapplied substances. Stable intracellular recordings from spinal cord neurons could be maintained for several hours. Only neurons with resting membrane potentials of $-40 \mathrm{mV}$ or more were used in experiments.

For assay of spontaneous or evoked postsynaptic potentials, hyperpolarizing and depolarizing currents were passed via the bridge circuit to augment amplitudes of excitatory (EPSPs) or inhibitory postsynaptic potentials (IPSPs), respectively, and facilitate accurate recognition above the noise level (noise $<200 \mu \mathrm{V}$ ).

Tetanus toxin dilutions in volumes equal to $25 \%$ of the final solution volume were prepared and premixed with the media on the cultures immediately prior to 
physiologic recordings in most experiments. At the higher concentrations of toxin (greater than $2 \times 10^{-7}$ $\mathrm{gm} / \mathrm{ml}$ ), experiments were also performed with addition of a similar volume to the cultures after stable intracellular recordings had been established. This allowed observations of the earliest toxin-produced phenomena.

Experiments with strychnine used $4 \times 10^{-4}$ to $10^{-6} \mathrm{M}$ of the $\mathrm{HCl}$ salt (buffered to $\mathrm{pH} 7.4$; Sigma Chemical Co., St. Louis, MO). Experiments were performed in the same manner as were the tetanus toxin experiments.

Iontophoretic application of GABA $(0.5 \mathrm{M}, \mathrm{pH} 3.5$; Sigma), glycine (0.5 M, pH 3.5; Sigma), and glutamic acid (0.5 M, pH 8.5; Sigma) used a constant current multichannel iontophoretic unit with head stage to apply the respective amino acids from micropipettes positioned near (1 to $2 \mu \mathrm{m}$ ) the neuronal cell membrane. Iontophoretic currents of 5 to $50 \mathrm{nA}$ and 50 to $200 \mathrm{msec}$ were used to elicit postsynaptic responses. Pressure application of tetanus toxin and strychnine was from micropipettes (with tips broken to 2 to $4 \mu \mathrm{m}$ diameter) in conjunction with a low pressure $\left(<1 \mathrm{~kg} / \mathrm{cm}^{2}\right)$ nitrogen gas system.
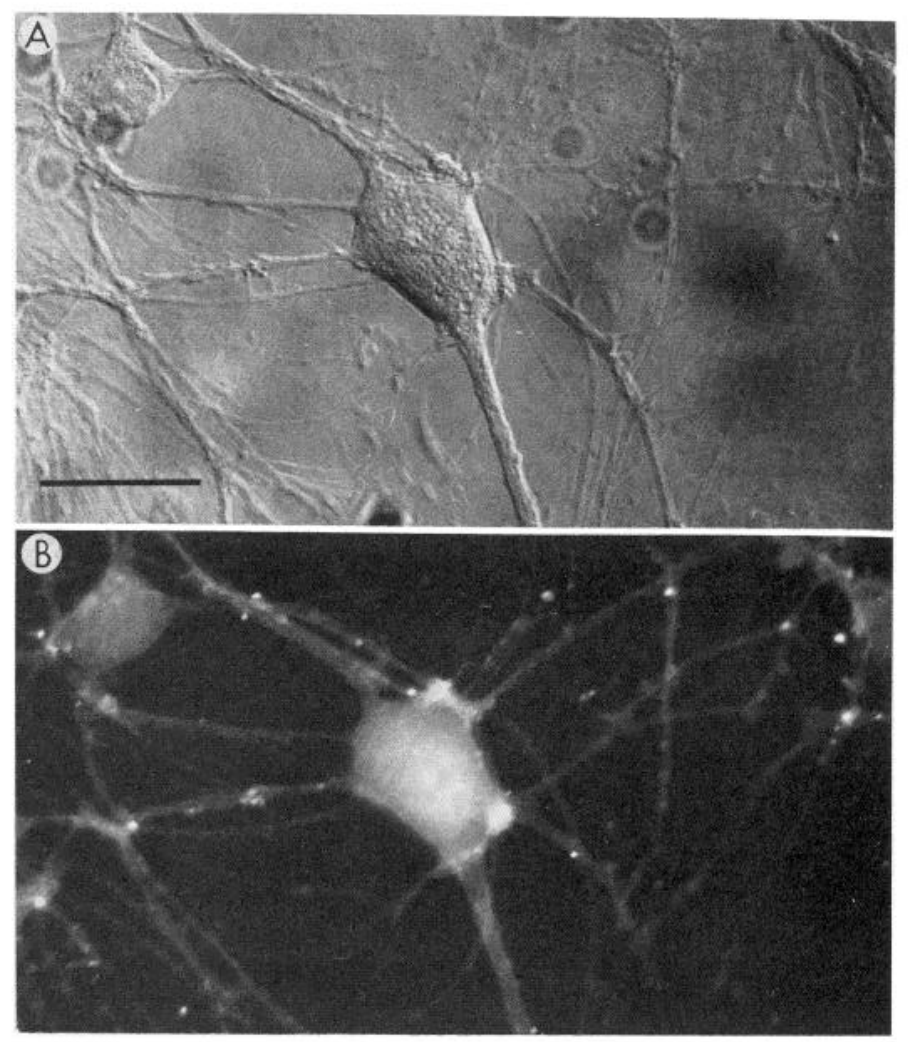

Figure 1. Tetanus toxin binds to spinal cord neurons. The upper photomicrograph $(A)$ illustrates spinal cord neurons in culture with fibroblasts and other neuronal elements as a supporting layer (Nomarski optics). The same microscopic field is shown in the lower photograph $(B)$ with epi-illumination and rhodamine fluorescence after application of $10^{-4} \mathrm{gm} / \mathrm{ml}$ of rhodamine-conjugated tetanus toxin for $15 \mathrm{~min}$ at $35^{\circ} \mathrm{C}$. Specific toxin binding to neuronal cell bodies and processes is demonstrated with only slight nonspecific binding to nonneuronal background cellular elements. Bar $=100 \mu \mathrm{m}$.

\section{Results}

Toxin binding to spinal cord neurons. Tetanus toxin directly conjugated with rhodamine was bound selectively to the spinal cord neurons in these cultures (Fig. 1). Rhodamine fluorescence of neuronal cell bodies and processes was prominent at toxin concentrations of $10^{-4}$ and $10^{-5} \mathrm{gm} / \mathrm{ml}$ after 10 to $60 \mathrm{~min}$ (maximum time tested). Incubation temperatures of $6^{\circ}$ or $35^{\circ} \mathrm{C}$ did not affect the degree of fluorescence observed. At toxin concentrations of 1 to $2.5 \times 10^{-5} \mathrm{gm} / \mathrm{ml}$ these direct staining methods produced a picture of stippled fluorescence over the neuronal cell bodies and processes. Whether this stippling represents localization to presynaptic structures remains to be determined. At higher toxin concentrations $\left(10^{-4} \mathrm{gm} / \mathrm{ml}\right)$ the fluorescent staining was more uniform in character. Preincubation $(1 / 2 \mathrm{hr})$ of the rhodaminated toxin with antitoxin blocked the specific neuronal binding. Specific binding of tetanus toxin to spinal cord neurons has been described previously using iodinated toxin (Dimpfel and Habermann, 1977) and indirect fluorescent techniques (Mirsky et al., 1978).

Tetanus-produced paroxysmal activity. When tetanus toxin was added to spinal cord cultures to yield final concentrations of $10^{-11}$ to $10^{-5} \mathrm{gm} / \mathrm{ml}\left(10^{-1}\right.$ to $10^{5} \mathrm{MLD} /$ $\mathrm{ml}$ ), the toxin regularly produced PDE (see below) in nearly all spinal cord neurons $(94 \% ; n=206$ for all toxin concentrations) sampled by intracellular recordings.

Intracellular recordings from spinal cord neurons under control conditions revealed prominent spontaneous activity consisting of postsynaptic potentials (excitatory and inhibitory) and action potentials. Addition of tetanus toxin produced no immediate effect on the spontaneous activity observed. After an initial lag period, however, the toxin produced increased excitatory activity that evolved into an organized pattern of PDE (Fig. 2). Preincubation $(1 / 2 \mathrm{hr})$ of tetanus toxin with excess TAT $(10$ $\mathrm{U} / 10^{-6} \mathrm{gm}$ of toxin) prior to addition to the cultures prevented the appearance of tetanus-PDE (Fig. 2). Strychnine $\left(10^{-6}\right.$ to $4 \times 10^{-4} \mathrm{M}$ final concentration) produced a pattern of paroxysmal depolarizations and bursts similar in appearance to tetanus-PDE; however, at all strychnine concentrations the onset of PDE was abrupt, usually occurring within $1 \mathrm{~min}$ (at most $10 \mathrm{~min}$ ) after the addition of strychnine (Fig. 2), in contrast to the long latency (always greater than $20 \mathrm{~min}$ ) to produce organized tetanus-PDE. At $0.1 \mathrm{mM}$, strychnine has approximately $4 \mathrm{MLD} / \mathrm{ml}$ (parenteral administration; Spector, 1956), whereas tetanus toxin at this concentration contains over $10^{8} \mathrm{MLD} / \mathrm{ml}$.

Another difference between the convulsant actions of strychnine and tetanus toxin was that the effects of strychnine were readily reversible, whereas those produced by tetanus toxin were not. Washing cultures in control medium to remove the strychnine abolished the convulsant action, and neurons returned to a control pattern of spontaneous activity. In contrast, washing toxin-treated cultures, even with control medium containing excess antitoxin, failed to eliminate the tetanusPDE after tbey had become established.

The tetanus-PDE (Fig. 3) were characterized by abrupt depolarizing shifts of 4 to $32 \mathrm{mV}$ (mean 14.0 $\pm 6.7 \mathrm{SD}$ ) 
CONTROL
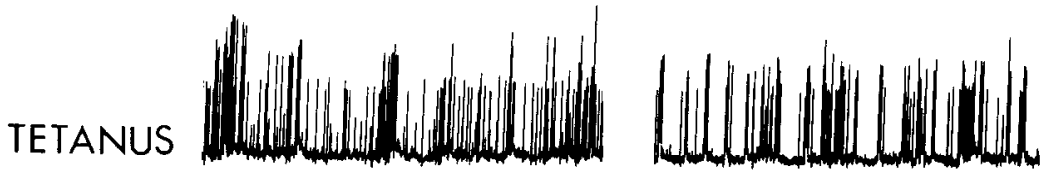
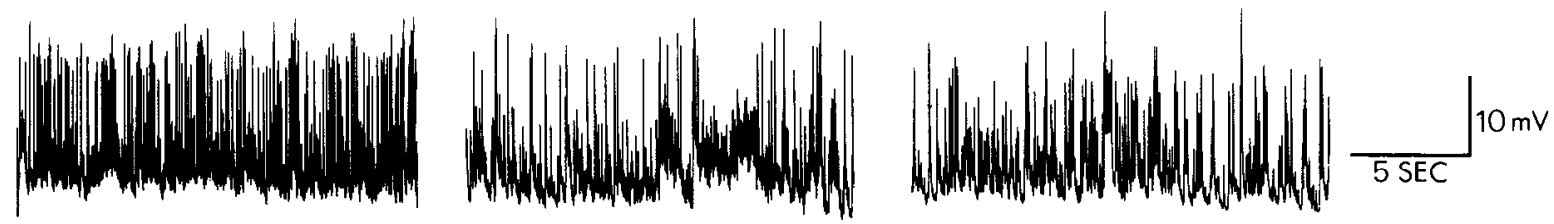

TETANUS
TÄT

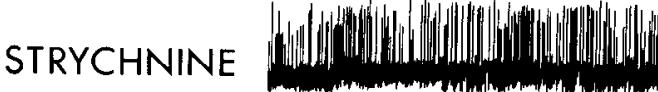
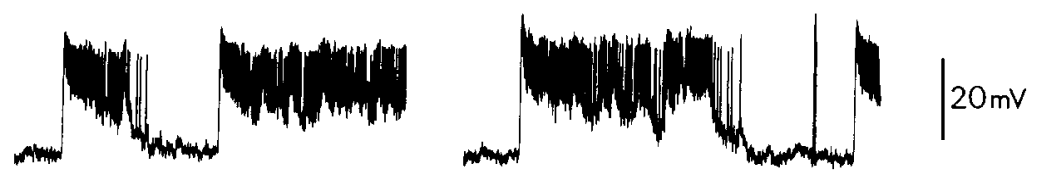

Figure 2. Convulsant action of tetanus toxin. Control penwriter chart recordings show a combination of spontaneous excitatory and inhibitory postsynaptic potentials with action potentials. In all chart records the limitations of the frequency response of the penwriter results in attenuation of action potential amplitudes. Addition of tetanus toxin $\left(7 \times 10^{-7} \mathrm{gm} / \mathrm{ml}\right.$ final concentration $)$ after a stable recording had been established resulted in no immediate change in observed spontaneous activity. However, recordings after $1 \mathrm{hr}$ in toxin reveal organized tetanus-PDE. Preincubation of tetanus toxin with TAT $(10 \mathrm{U} / \mathrm{ml})$ before addition to the cultures prevented the convulsant effects of the toxin. In contrast to tetanus, strychnine $(0.1 \mathrm{~mm}$ final concentration) produced rapid onset of paroxysmal activity wilhin minutes after addition to the cultures.
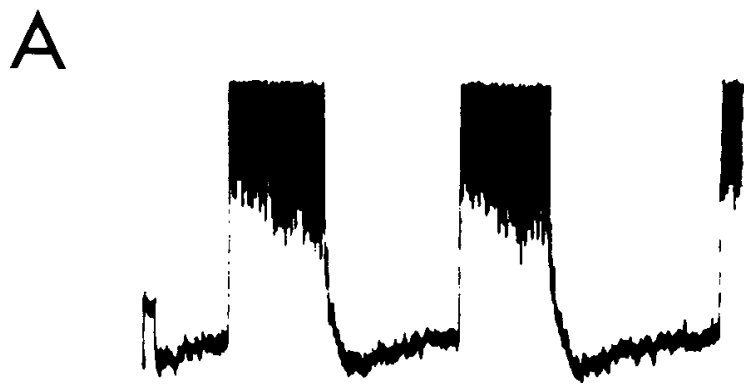

B
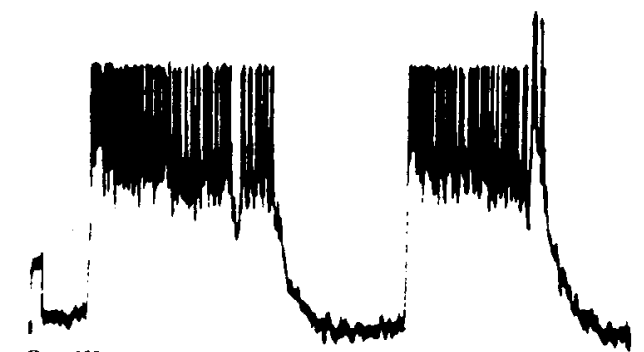

Figure 3. Oscilloscope records of tetanus toxin-produced depolarizing events. Shown are recordings from neurons in $10^{-7}$ $\mathrm{gm} / \mathrm{ml}$ of tetanus toxin after $100 \mathrm{~min}(A)$ and $120 \mathrm{~min}(B)$. Although some premonitory spike and synaptic activity can be seen (Fig. 11), frequently, as illustrated here, the tetanus-PDE were characterized by abrupt depolarizing shifts with resultant triggered action potentials. In some instances (see the text) the PDE were followed by a hyperpolarization before return to resting membrane potential $(A)$. More often, no discrete AHP could be seen $(B)$. $B$ shows a superimposed second small PDE on the large PDE. Both recordings shown are at resting membrane potential $(-56 \mathrm{mV}$ in $A,-60 \mathrm{mV}$ in $B)$. The calibration pulse is $10 \mathrm{mV}, 200 \mathrm{msec}$. from resting membrane potential with a resulting train of action potentials on a depolarization plateau lasting $200 \mathrm{msec}$ to as long as $11 \mathrm{sec}$, with $84 \%$ of one sample $(n=100)$ having depolarizations of greater than 1000 msec before returning to resting membrane potential. The frequency of PDE observed ranged from 2 to 54/ min. In some neurons (22\%) the tetanus-PDE at resting membrane potential were followed by hyperpolarizations of 1 to $4 \mathrm{mV}$ for 1 to $2 \mathrm{sec}$. Most neurons with tetanusPDE, however, did not exhibit demonstrable after-hyperpolarizations (AHP), and at times the AHP disappeared as the PDE evolved over hours. With the longerduration PDE, some inactivation of the spike-generating mechanism was frequently observed during the plateau of depolarization.

A small percentage (about 5\%) of control spinal cord neurons exhibited bursting behavior, at times associated with depolarizing shifts. These spontaneous bursts were less organized and of shorter duration (100 to $300 \mathrm{msec})$ than the PDE produced by tetanus; such neurons were not used in determining the onset of tetanus convulsant action.

The time of onset of established tetanus-PDE after the addition of toxin was dose dependent, ranging from an average of $26 \mathrm{~min}$ at $10^{-6} \mathrm{gm} / \mathrm{ml}$ of toxin to $523 \mathrm{~min}$ at $10^{-11} \mathrm{gm} / \mathrm{ml}$ (Fig. 4). A toxin concentration of $10^{-11}$ $\mathrm{gm} / \mathrm{ml}\left(10^{-1} \mathrm{MLD} / \mathrm{ml}\right)$ was the lowest that would reliably produce PDE in the neurons sampled ( $90 \%$ of cells). Only $10 \%$ of neurons demonstrated PDE within $48 \mathrm{hr}$ in tetanus toxin at $10^{-12} \mathrm{gm} / \mathrm{ml}$. No neurons developed PDE when exposed to tetanus toxin at $10^{-13} \mathrm{gm} / \mathrm{ml}$ for up to 4 days. The possibility exists that, at these lowest toxin concentrations, toxin could be neutralized by antitoxin from residual horse serum. Our bioassay for residual 


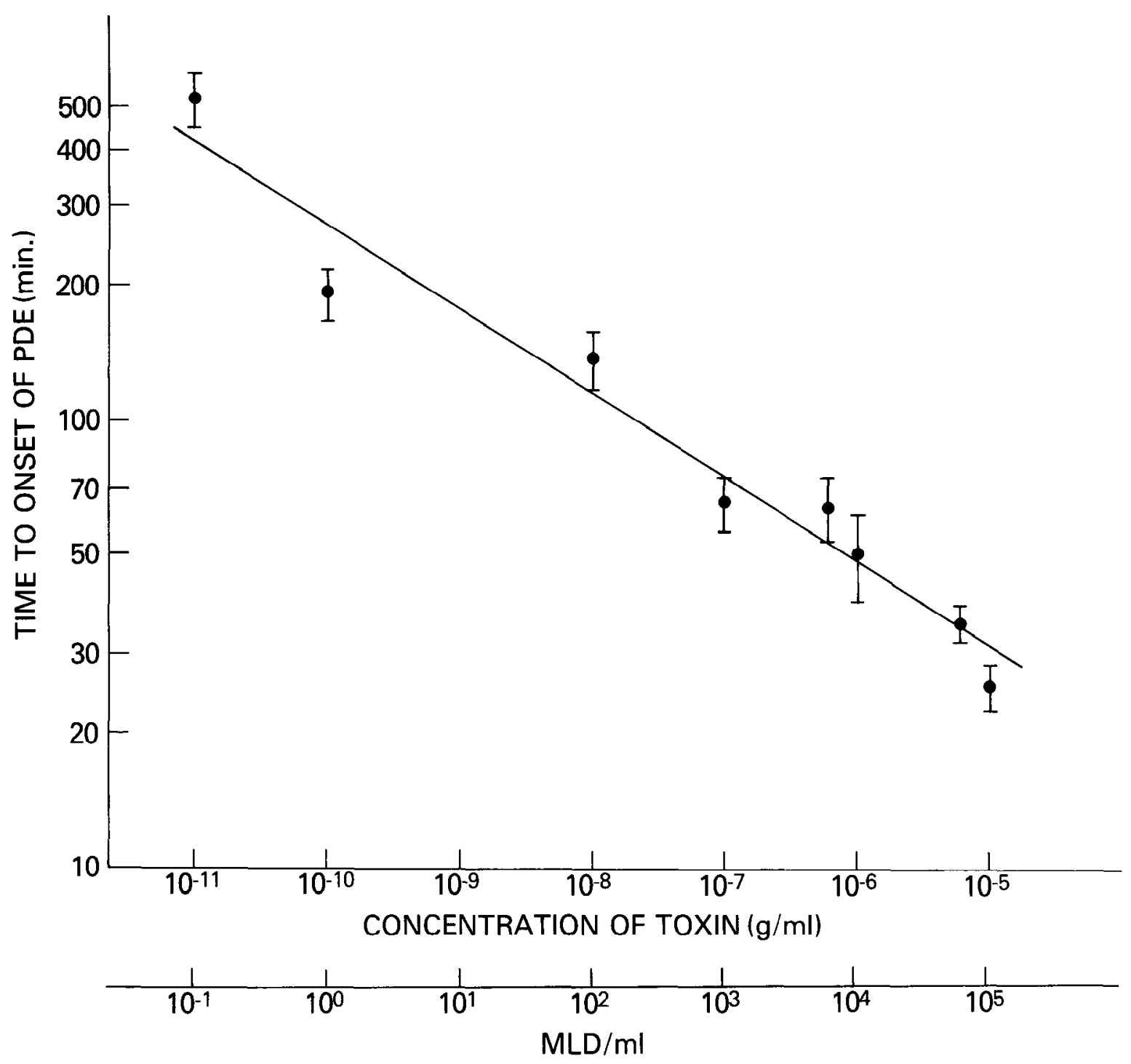

Figure 4. Time-dependent lag to onset of tetanus toxin-produced PDE. Shown is the time of onset of PDE as a function of concentration of tetanus toxin. Each point $( \pm \mathrm{SEM})$ represents the average of three to eight separate timed experiments at each toxin concentration. The second abscissa shows the number of mouse lethal doses (MLD) per milliliter for each concentration.

$$
11 \mathrm{hr} 10^{-11} \mathrm{~g} / \mathrm{ml} \text { TETANUS }
$$

A

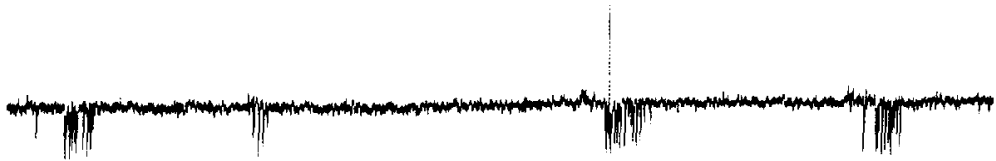

B

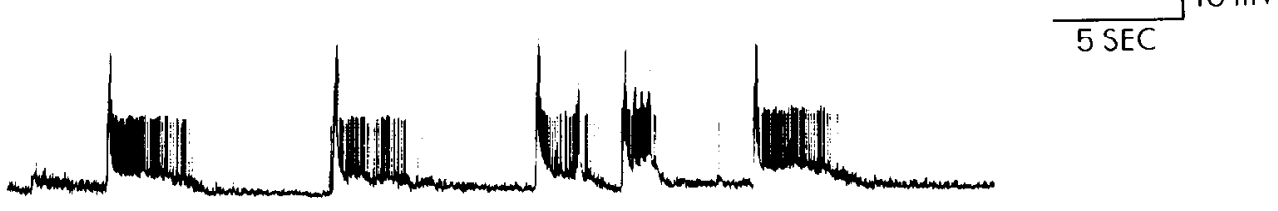

Figure 5. Chart records from two spinal cord neurons in the same culture after $11 \mathrm{hr}$ of exposure to low dose tetanus toxin $\left(10^{-11} \mathrm{gm} / \mathrm{ml}\right)$. Cell $A$ illustrates one of the few spinal cord neurons that did not develop tetanus-PDE. IPSPs are the predominant spontaneous activity observed and are quite prominent; there are few EPSPs. Cell $B$ illustrates characteristic tetanusPDE in a cell from a different area of the same culture. Amplitudes of action potentials are attenuated by the limited frequency response of the penwriter. 
A CONTROI

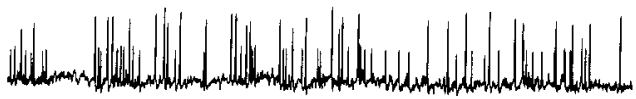

TETANUS $\left(10^{-8} \mathrm{~g} / \mathrm{ml}\right)$
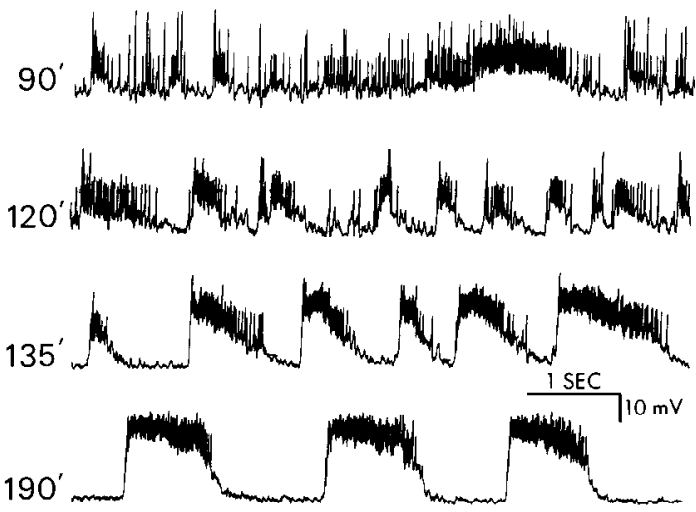

B

INTER-PDE ACTIVITY
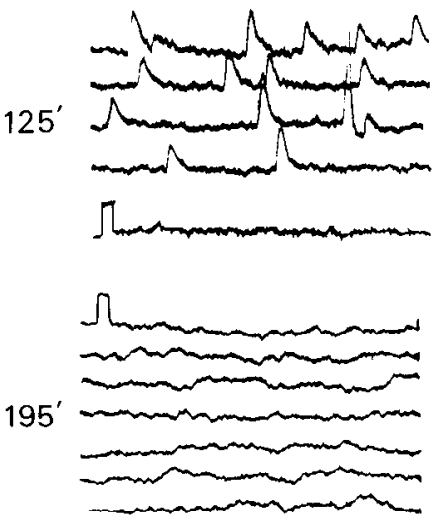

Figure 6. Evolution of tetanus toxin-produced PDE. Chart records $(A)$ from a continuous intracellular recording from a spinal cord neuron under control conditions at various intervals after addition of an intermediate concentration $\left(10^{-8} \mathrm{gm} /\right.$ $\mathrm{ml}$ ) of tetanus toxin. At these lower concentrations the onset of PDF is less abrupt; the chart records reveal the gradual increase in excitatory activity until, in the later records, the PDE are well established. As illustrated at 190 min the PDE often have a quite regular periodicity. Oscilliscope sweeps of the inter-PDE intervals are shown in $B$. Early in PDE synaptic activity is frequently seen in the inter-PDE intervals, but as the PDE becomes more organized (in $A$ ), synaptic events are clustered near the PDE, and the inter-PDE intervals often show few spontaneous synaptic potentials (195 $\mathrm{min}$ in $B$ ). The calibration pulse in $B$ is $5 \mathrm{mV}, 10 \mathrm{msec}$.

antitoxin is sensitive only to $10^{-3} \mathrm{U}$, thus lesser amounts of antitoxin could remain undetected after repeated washings. Only $10^{-6} \mathrm{U}$ of residual antitoxin/culture would be necessary to neutralize the toxin at $10^{-12} \mathrm{gm} /$ ml. In preliminary experiments (G. K. Bergey and $H$. Bigalke, unpublished data) with cultures grown primarily in serum-free medium (modified N2 medium, Bottenstein and Sato, 1979), $10^{-12} \mathrm{gm} / \mathrm{ml}$ of toxin $\left(10^{-2} \mathrm{MLD} /\right.$ $\mathrm{ml}$ ) regularly produced $\mathrm{PDE}$.

There was some variation in the time of onset of organized tetanus-PDE at all concentrations of toxin used (Fig. 4). The most rapid onset (20 min) was observed at the highest toxin concentration shown $\left(10^{-5} \mathrm{gm} / \mathrm{mll}\right)$ in a cell that exhibited no spontaneous IPSPs and had a control pattern of frequent bursts of action potentials (without depolarizing shifts). A single experiment at a toxin concentration of $5 \times 10^{-5} \mathrm{gm} / \mathrm{ml}$ demonstrated a latency of $25 \mathrm{~min}$ before $\mathrm{PDE}$, supporting an apparent obligatory lag period in contrast to the rapid action of strychnine. Higher concentrations of tetanus toxin were not tested.

At the higher toxin concentrations $\left(10^{-7}\right.$ to $10^{-5} \mathrm{gm} /$ $\mathrm{ml}$ ), once PDE developed in one neuron they were found to be present in other neurons subsequently sampled. At lower toxin concentrations $\left(10^{-10}\right.$ and $\left.10^{-11} \mathrm{gm} / \mathrm{ml}\right)$, however, some variability in latencies to onset of tetanusPDE could be found in different neurons. The longest latencies were seen in those cells with the most prominent spontaneous inhibitory synaptic input and/or reduced excitatory synaptic activity. For example, using a very low toxin concentration $\left(10^{-11} \mathrm{gm} / \mathrm{ml}\right)$, one cell had
PDE and another remote neuron with prominent IPSPs had not yet developed PDE (Fig. 5). In this instance the IPSPs present were of long duration. The few neurons (6\%) that failed to demonstrate PDE were often relatively isolated from other neurons in the culture and failed to exhibit prominent excitatory synaptic activity in the presence of tetanus toxin. Within the range of toxin concentrations reported $\left(10^{-11}\right.$ to $\left.10^{-5} \mathrm{gm} / \mathrm{ml}\right)$, failure to show tetanus-PDE was not dose dependent. Although a broad range of tetanus toxin concentrations produced the characteristic PDE, most of the reported experiments were done using toxin concentrations of $10^{-7}$ to $10^{-6} \mathrm{gm} / \mathrm{ml}$. At these loxin concentrations the evolution of tetanus PDE occurred over one to several hours, permitting continuous recording from a given neuron throughout, under favorable circumstances. At lower toxin concentrations, the evolution and characteristics of the PDE were similar; however, evolution required a considerably longer time, making continuous recording from a single neuron technically more difficult.

Synaptic activity during tetanus-PDE. An intermediate toxin concentration $\left(10^{-8} \mathrm{gm} / \mathrm{ml}\right)$ produced a more gradual evolution of excitatory events (Fig. 6). As described above, a dose-dependent latency was present. The first changes in spontaneous activity were increases in spontaneous action potentials with clustering and bursting. At the time of onset of organized tetanus-PDE (120 min in Fig. 6), the inter-PDE intervals exhibited considerable spontaneous activity. With further evolution the tetanus-PDE became quite regular (frequency range, 3 to 40 / min for all cells). Concomitant with this increased peri- 
odicity and organization, the inter-PDE intervals became increasingly quiescent (Fig. 6B).

Although IPSPs were observed in the early stages of some spinal cord neurons with tetanus-PDE, the incidence of neurons exhibiting detectable (using membrane depolarization to augment IPSP amplitude) IPSPs in early tetanus-PDE was reduced to $25 \%$ of control (to 6 of 50 versus control of 24 of 50 , Table I). In contrast, the number of spinal cord neurons demonstrating spontaneous EPSPs increased slightly (13\%) over control (to 44 of 50 versus control of 39 of 50 ). In all neurons where recordings of sufficient duration were maintained, spontaneous inhibitory synaptic potentials diminished in amplitude and disappeared (Fig. 7), whereas PDE and demonstrable excitatory synaptic poentials persisted.

In contrast to the early convulsant action produced by tetanus toxin, continued exposure to toxin concentrations of $10^{-5}$ to $10^{-10} \mathrm{gm} / \mathrm{ml}$ resulted in the ultimate disappearance of all spontaneous synaptic acitivty after a dose-dependent interval (range, 2 to $12 \mathrm{hr}$ ). After these times paroxysmal depolarizations were no longer seen. The disappearance of PDE with chronic exposure to tetanus coincided with blockade of all synaptic activity

\section{TABLE I}

Observed spontaneous synaptic activity

The incidence of spontaneous EPSPs and IPSPs in control spinal cord neurons and in spinal cord neurons after tetanus toxin $\left(10^{-7}\right.$ to $10^{-6} \mathrm{gm} / \mathrm{ml}$ ) had produced PDE demonstrates a reduction in observed inhibitory synaptic activity. An increase in the frequency of EPSPs accompanied the diminished inhibitory activity. Observations were done with membrane hyperpolarization and depolarization with constant current to accentuate EPSPs and IPSPs, respectively. Sampling after tetanus treatment was performed soon after the onset of tetanusPDE ( 1 to $1^{1 / 2} \mathrm{hr}$ ). Spinal cord neurons exhibiting PDE after tetanus toxin exposure were included in the tetanus sample. Only unequivocal postsynaptic potentials were counted; some neurons exhibiting PDE did not have discernible discrete EPSPs separate from the PDE, although evidence indicates that the PDE are mediated by excitatory synaptic phenomena (see the text).

\begin{tabular}{lcr}
\hline & EPSPs & \multicolumn{1}{c}{ IPSPs } \\
Control Spinal Cord Neurons $(n=50)$ & $39(78 \%)$ & $24(48 \%)$ \\
Tetanus Toxin Treated (early PDE) $(n$ & $44(88 \%)$ & $6(12 \%)$ \\
$\quad=50)$ & &
\end{tabular}

by the toxin (Bergey et al., 1981). Strychnine-treated cultures continued to demonstrate characteristic depolarizing bursts after $24 \mathrm{hr}$ of exposure. At the lowest convulsant dose of tetanus toxin $\left(10^{-11} \mathrm{gm} / \mathrm{ml}\right)$, PDE were still observed in neurons after 4 days of continuous exposure to toxin.

Synaptic mechanisms of tetanus-PDE. All simultaneous recordings from cell pairs in the same microscopic field after tetanus toxin addition revealed synchrony of the observed PDE in both cells. This was evident whether or not the cells had demonstrable evoked synaptic connections (Figs. 8, $A$ and $B$ ). Neuronal groups remote from each other in a given culture, however, often had different PDE frequencies.

DRG neurons in these cultures, although able to form synapses with spinal cord neurons, have few, if any, synaptic inputs themselves and exhibit no evidence of spontaneous synaptic activity (Ransom et al., 1977c). Recordings from DRG neurons $(n=15)$ after tetanus toxin addition revealed no evidence of PDE or change in spontaneous activity at a time when nearby spinal cord neurons demonstrated well established PDE (Fig. 8C).

Addition of $10 \mathrm{~mm}$ magnesium to the spinal cord cultures abolished tetanus-PDE (Fig. 9A). This concentration of magnesium was sufficient to block observed spontaneous synaptic transmission in these cultures (Ransom et al., 1977b), although some quantal release of transmitter may still have occurred (del Castillo and Katz, 1954; Katz and Miledi, 1963). Addition of tetrodotoxin (TTX), which blocks voltage-dependent sodium conductance (see review by Evans, 1972) to the spinal cord neurons after tetanus-PDE had developed, abolished PDE as well as eliminating action potentials. Blockade of action potentials effectively eliminated stimulated transmitter release (i.e., postsynaptic potentials). In the presence of TTX no TTX-resistant intrinsic components of the depolarizing shift were observed (Fig. $9 B)$.

In spinal cord cultures where tetanus-PDE had developed, stimulation of single neurons with superthreshold ( 0.5 to $3 \mathrm{nA}, 10$ to $200 \mathrm{msec}$ ) depolarizing pulses failed to reliably trigger PDE (Fig. 9C). Similarly, stimulation of the presynaptic cell of a monosynaptic excitatory cell

\section{TETANUS $\left(10^{-10} \mathrm{~g} / \mathrm{ml}\right)$}
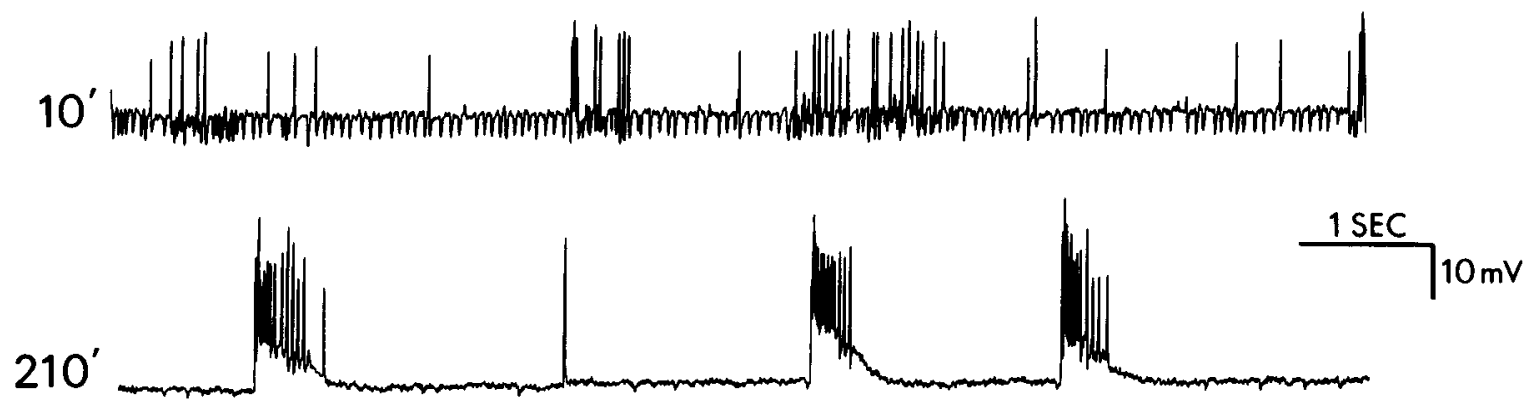

Figure 7. Reduction of inhibition with development of tetanus-PDE. Selected chart records of a continuous recording from a spinal cord neuron are shown after the addition of tetanus toxin $\left(10^{-10} \mathrm{gm} / \mathrm{ml}\right)$. At 10 min multiple spontaneous IPSPs are present. After $210 \mathrm{~min}$ of exposure to toxin, shortly after the appearance of PDE, spontaneous IPSPs are much less prominent, even with membrane depolarization (not shown). Resting membrane potential was $-56 \mathrm{mV}$ at $10 \mathrm{~min},-54 \mathrm{mV}$ at $210 \mathrm{~min}$. 


\section{A}

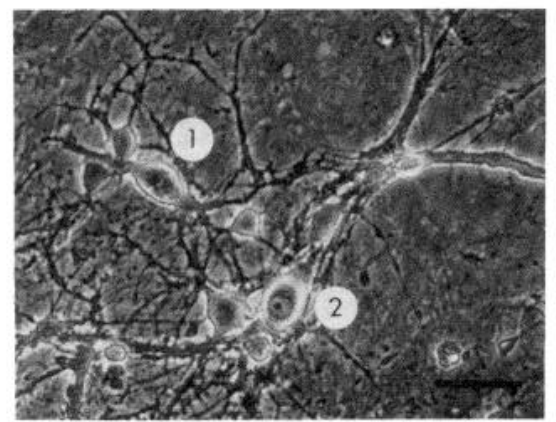

B

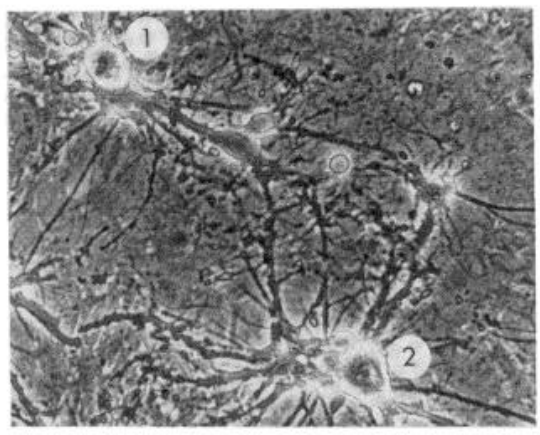

C

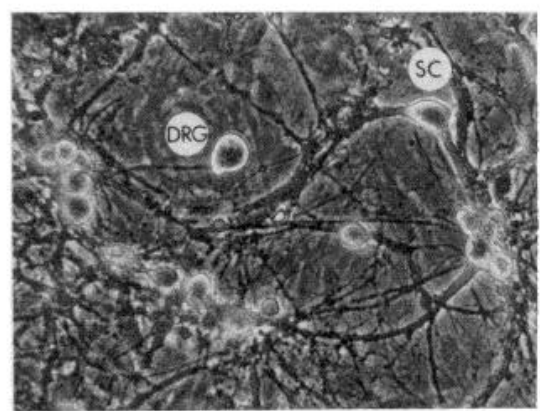

\section{1-2 EPSP}

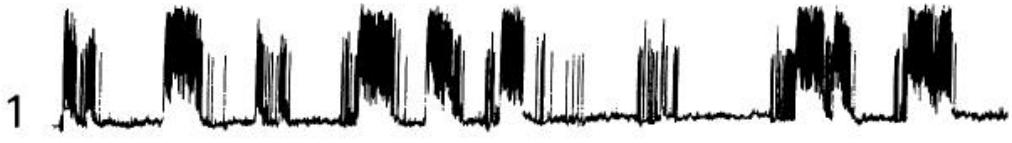

$2 H^{2}$
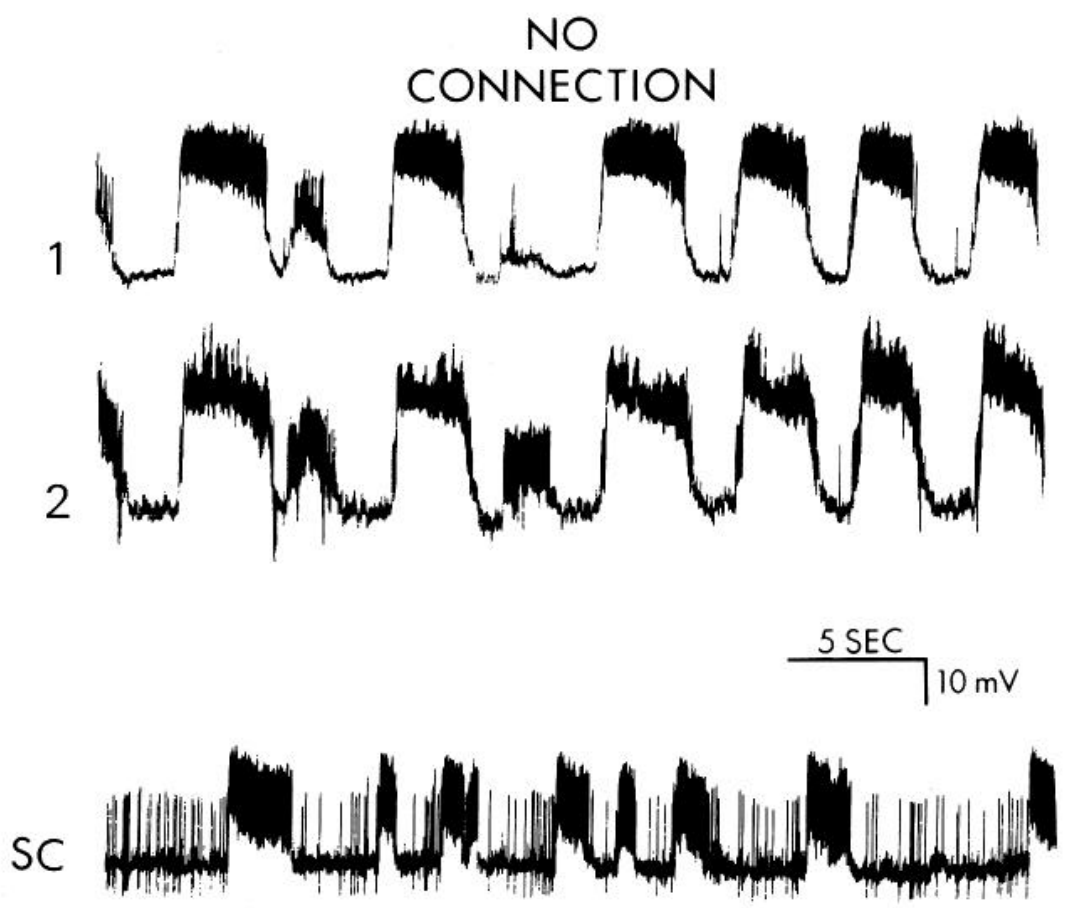

DRG

Figure 8. Tetanus produces synchronous PDE in cell pairs. Photomicrographs (calibration bar $=50 \mu \mathrm{m}$ ) of the recording field are shown to the left of penwriter records of simultaneous recordings from the illustrated cell pair. In $A$, cell 1 was demonstrably connected to cell 2 by a monosynaptic EPSP. The tetanus toxin-produced PDE were synchronous $\left(10^{-7}\right.$ gm/ml toxin after 110 $\mathrm{min})$. In $B$, no demonstrable synaptic connection between cells 1 and 2 were present; spontaneous synaptic activity was prominent in the control situation. PDE produced by tetanus toxin were synchronous in both cells. $C$ shows a spinal cord ( $S C$ ) neuron with PDE produced by tetanus toxin $\left(10^{-7} \mathrm{gm} / \mathrm{ml}\right)$. An adjacent dorsal root ganglion $(D R G)$ neuron demonstrates no convulsant effects of the toxin $120 \mathrm{~min}$ after toxin addition.

pair could not reliably trigger the PDE in either cell. In a few instances (six cells) the frequency of tetanus-PDE could be increased by rapid ( 4 to $10 \mathrm{~Hz}$ ) superthreshold stimulation of the spinal cord neuron (Fig. 9D); such observed increases in PDE frequency probably resulted from increased synaptic activity since direct triggering of PDE with low frequency depolarizing pulses did not occur. The clustered and, at times, superimposed PDE (Fig. $3 B$ ) suggest that the inter-PDE period is not an absolutely refractory period to $\mathrm{PDE}$ generation. In a very few cells $(n=3)$ direct stimulation could evoke depolarizing shifts, but these events were relatively brief (100 to $200 \mathrm{msec}$ duration) relative to the longer spontaneous tetanus-PDE. 

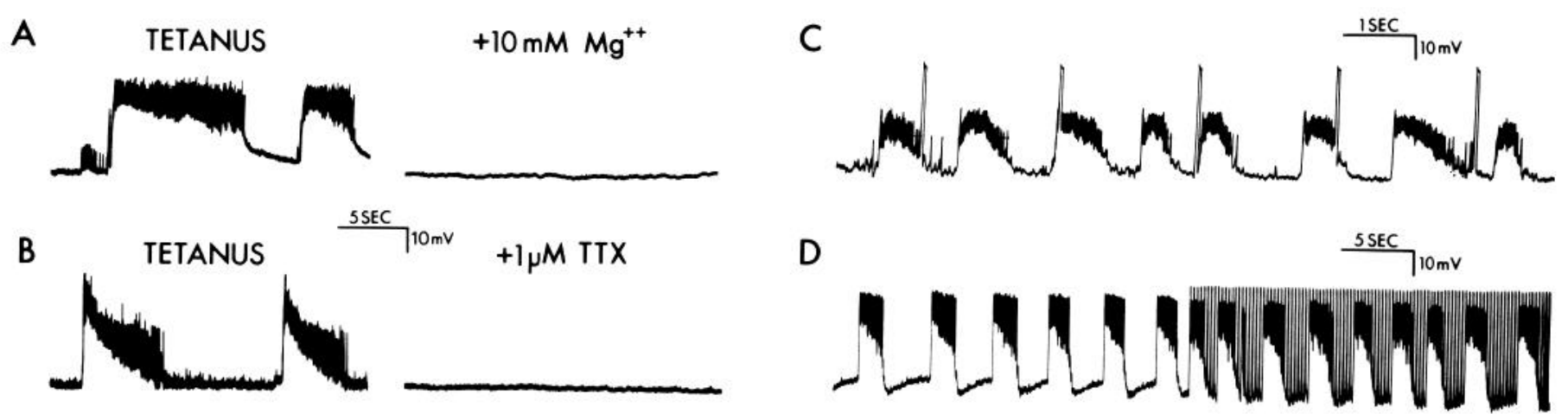

Figure 9. Addition of magnesium (final concentration $10 \mathrm{~mm}$ ) to the cultures abolished the tetanus-PDE $(A)$. Similarly, addition of tetrodotoxin $(T T X)$ also eliminated the PDE $(B)$; no underlying intrinsic membrane depolarizations were revealed. The depolarizing events could not be triggered by direct stimulation $(C)$, although rapid $(4 \mathrm{~Hz})$ intracellular suprathreshold stimulation (4 nA, 50-msec pulses) at times increased the PDE frequency $(D)$, presumably by synaptic mechanisms.

$-56 \mathrm{mV}$

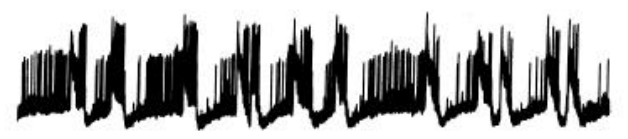

$-70 \mathrm{mV}$

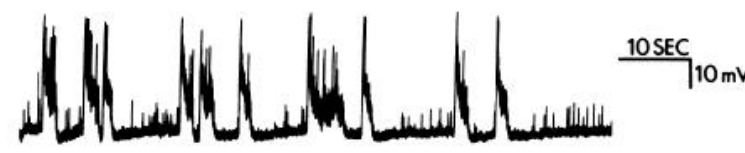

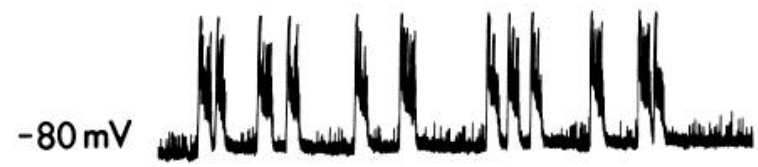

Figure 10. Hyperpolarization of the neuronal membrane by passage of constant negative DC current had no effect on the frequency of tetanus-PDE. Some triggered action potentials were abolished by hyperpolarization; their underlying EPSPs were revealed. The amplitude of the depolarizing shift increased with hyperpolarization and the AHP flattened at $-80 \mathrm{mV}$.

Hyperpolarization of the neuronal membrane of spinal cord neurons in culture reduced the frequency of intrinsic action potentials. In instances of synaptically triggered action potentials, the passage of constant hyperpolarizing current also typically reduced the spike frequency but in so doing unmasked the underlying excitatory synaptic potentials. Hyperpolarization of spinal cord neurons demonstrating tetanus-PDE (to -90 to -100 $\mathrm{mV}$ membrane potential) did not reduce PDE frequency (Fig. 10). In most neurons the depolarizing shifts were of sufficient magnitude that action potentials continued to be present in the PDE. In a few instances of tetanusPDE of lesser magnitude, constant hyperpolarization reduced the triggered action potentials and revealed a depolarizing shift with EPSPs on the plateau (Fig. 11, A and $B$ ) without affecting the PDE frequency.

The PDE produced by tetanus frequently ( $37 \%$ of a sample of 100) were abrupt shifts from resting membrane potential without discernible initiating events (Figs. 3 and $11 A$ ). However, the shift often $(63 \%)$ was visibly preceded by increased excitatory activity, either action potentials or EPSPs (Fig. 11C). The illustrated neuron shows multiple EPSPs preceding and coincident with the onset of the depolarizing shift.
Using single intracellular microelectrodes with bridge circuits to inject current and record potentials, the extrapolated reversal potential of the depolarizing event was $+9 \pm 14 \mathrm{mV}$ (SD; $n=13$ ). A plot from one such cell is illustrated (Fig. 12).

Tetanus toxin had no demonstrable effect on the postsynaptic responses to iontophoretically applied amino acid neurotransmitters. Maximum responses for a given iontophoretic current at a selected region of the cell soma or proximal processes were elicited to minimize the possible limitations of the iontophoretic technique over a 2$\mathrm{hr}$ continuous period during which the PDE became well established. In some cultures $10 \mathrm{mM}$ magnesium was added subsequently to eliminate the PDE and other synpatic events for clearer postsynaptic responses. No demonstrable attenuation of postsynaptic iontophoretic responses to the inhibitory amino acids glycine $(n=6)$ and GABA $(n=5)$ were observed (Fig. 13A). The iontophoretic response to glutamate $(n=5)$, an excitatory amino acid, was similarly unaffected by tetanus (not shown). In contrast, cells in $100 \mu \mathrm{M}$ strychnine had no elicitable postsynaptic responses to iontophoretically applied glycine (maximal iontophoretic current $100 \mathrm{nA}, 200$ msec).

Direct pressure application of strychnine $(100 \mu \mathrm{M})$ via micropipette rapidly and reversibly abolished the postsynaptic response to iontophoretically applied glycine in normal medium (Fig. 13B). Similar application of tetanus toxin $(1 \mu \mathrm{g} / \mathrm{ml})$ had no effect on glycine responses (Fig. 13B). Direct pressure application of tetanus toxin by micropipette had no acute effect on resting membrane potential or membrane conductance $(n=5)$.

\section{Discussion}

Application of tetanus toxin to mouse spinal cord neurons in culture reliably produces PDE with spike bursts. The convulsant effects of tetanus toxin are seen at concentrations as low as $10^{-11}$ to $10^{-12} \mathrm{gm} / \mathrm{ml}\left(10^{-1}\right.$ to $10^{-2} \mathrm{MLD} / \mathrm{ml}$ ). Although it is difficult to equate these in vitro toxin concentrations with the amount of toxin that ultimately reaches the ventral cord after peripheral in vivo injection or infection, nevertheless, spinal cord neurons in culture are quite sensitive to effects of small amounts of toxin.

These spinal cord cultures contain a heterogeneous 

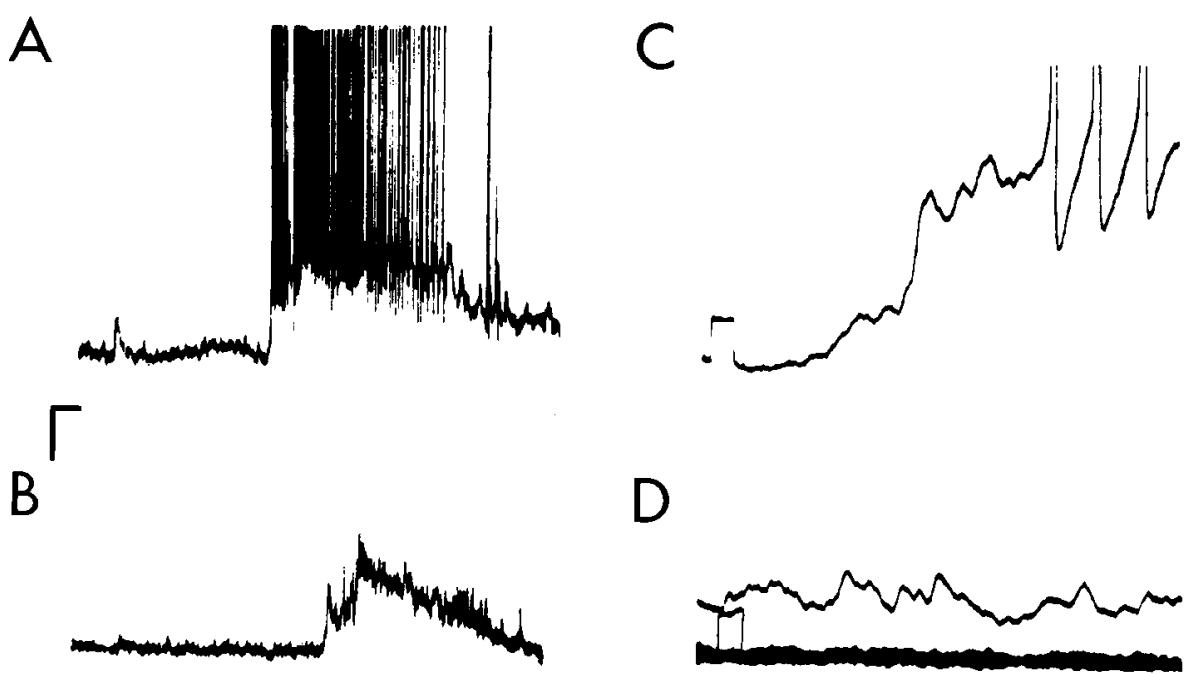

Figure 11. Excitatory synaptic events and tetanus-PDE. An oscilloscope photograph $(A)$ at resting membrane potential $(-55 \mathrm{mV})$ illustrates tetanus-PDE produced in a spinal cord neuron after exposure to tetanus toxin $\left(10^{-7} \mathrm{gm} / \mathrm{ml}\right)$. The characteristic membrane shift and triggered action potentials are seen. In this case, perhaps because of the comparatively small depolarizing shift present, hyperpolarization (to $-65 \mathrm{mV}$ ) of the membrane $(B)$ with constant negative DC current eliminated the action potentials while the depolarizing shifts persisted. Unmasked and amplified by this membrane hyperpolarization were apparent EPSPs that immediately preceded the PDE and were present on the crest of depolarizing shift. A more rapid sweep speed illustrates these EPSPs $(D)$ on the upper trace. The lower trace is 12 successive sweeps in the inter-PDE period demonstrating the paucity of synaptic activity in these periods. $C$ illustrates a recording from a different spinal cord neuron at high gain. Shown is the apparent summation of EPSPs leading to the depolarizing shift. The action potentials are clipped in all cases by the high oscilloscope gains. The calibrations are $10 \mathrm{mV}, 500 \mathrm{msec}$ for $A$ and $B, 5 \mathrm{mV}, 10 \mathrm{msec}$ in $C$, and $5 \mathrm{mV}, 5 \mathrm{msec}$ in $D$.

neuronal cell population including interneurons, motoneurons, and DRG neurons. Some of the large multipolar neurons appear morphologically similar to identified motoneurons in the intact ventral horn (Ransom et al., $1977 \mathrm{~b})$ and are capable of innervating muscle explants (Giller et al., 1973). Density gradients of rat spinal cord cells have demonstrated that the large cell fractions are highly enriched for neurons that produce choline acetyltransferase, the enzyme responsible for acetylcholine synthesis (Schnarr and Schaffner, 1981). Therefore, the subpopulation of large neurons sampled physiologically here appears to include the motoneurons important in the pathophysiology of tetanus.

The time required for tetanus toxin to produce paroxysmal activity in cultured spinal cord neurons is dose dependent. Even at the highest concentrations of toxin tested, there is an obligatory latent period of 20 to 30 min before the onset of the organized paroxysmal activity. This is in contrast to the rapid onset of convulsant action produced by the convulsant strychnine, which antagonizes the postsynaptic inhibitory action of glycine (Curtis et al., 1968, 1971; Davidoff et al., 1969). Other convulsants that act by antagonism of GABAergic postsynaptic inhibition (e.g., penicillin, bicuculline, and picrotoxin) also produce prompt effects in cultured spinal cord neurons (Macdonald and Barker, 1978; Heyer et al., 1981b).

The binding of tetanus toxin to spinal cord neurons is to a great degree irreversible (Dimpfel and Habermann, 1977) and may represent a complicated membrane interaction or internalization of toxin (Yavin et al., 1981). Gangliosides, particularly di- and trisialogangliosides, have been demonstrated to bind tetanus toxin (Van Heyningen, 1974; Dimpfel et al., 1977; Ledley et al., 1977), but whether these gangliosides represent the functional site of action of the toxin is disputed (Bigalke et al., 1981a; Mellanby and Green, 1981). Once established in cultured spinal cord neurons, the convulsant action of tetanus toxin is also irreversible. The latency required for onset of tetanus convulsant action may reflect a specific receptor-mediated internalization process.

The results presented here are consistent with a presynaptic locus of action for tetanus toxin. No alterations in responsiveness to iontophoretically applied glycine or GABA are produced by the toxin. In intact spinal cord, a reduction by tetanus toxin of monosynaptic inhibition is presumed to be by a presynaptic mechanism (Brooks et al., 1957); no reduction in neuronal sensitivity to glycine is found (Curtis and DeGroat, 1958; Guschin et al., 1969). At the neuromuscular junction, tetanus toxin produces a presynaptic blockade of transmission (Mellanby and Thompson, 1972; Habermann et al., 1980). The morphological studies mentioned in the introduction report a presynaptic localization of the toxin.

The excitation and paroxysmal depolarizations pro- 


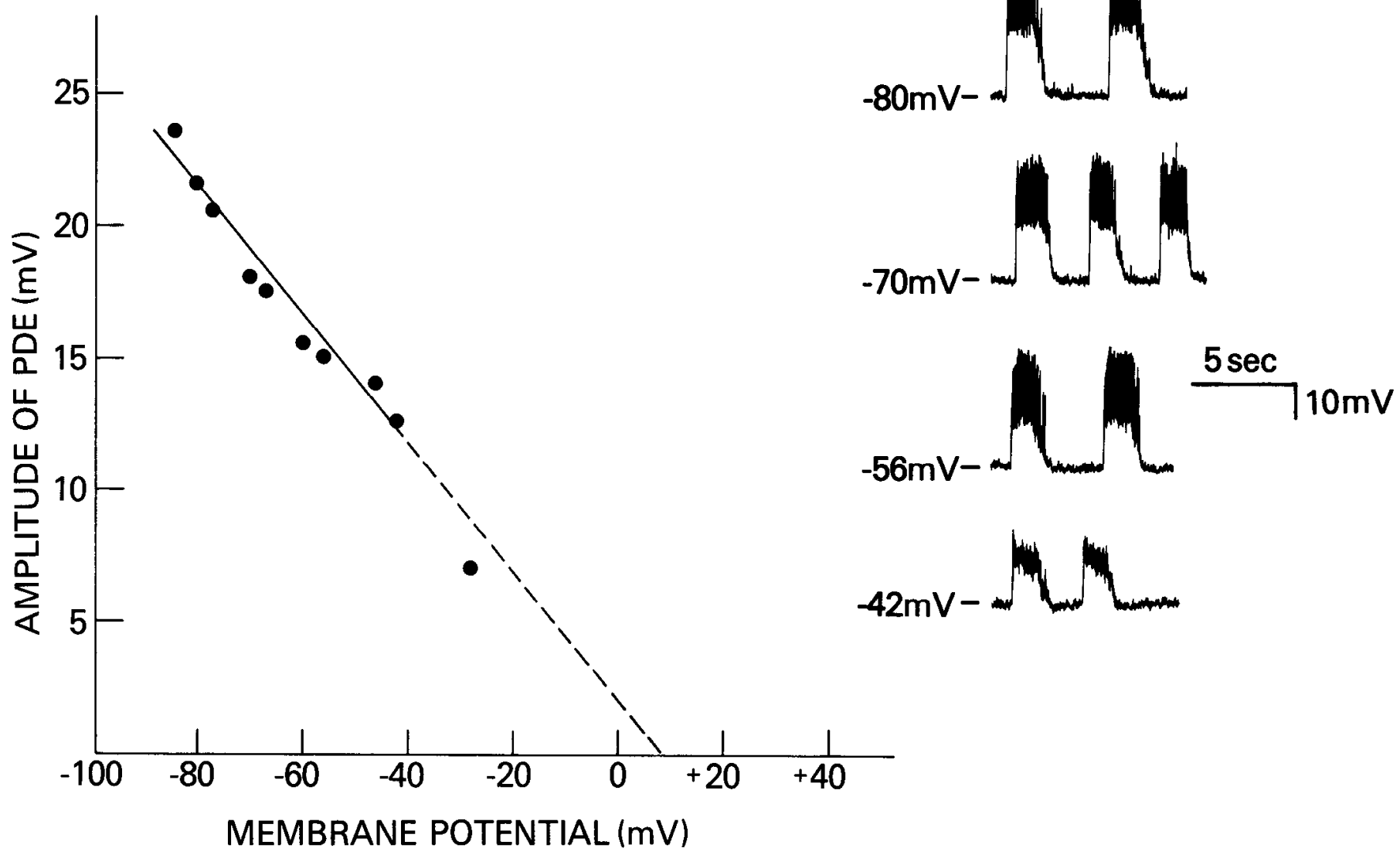

Figure 12. Reversal potential of depolarizing shift. The amplitude of the initial component of the depolarizing shift produced by tetanus toxin was extrapolated to a reversal potential of $+8 \mathrm{mV}$ in the case illustrated. Constant DC current was passed through the recording electrode to provide the desired membrane hyperpolarization or depolarization with careful attention to bridge balance. The point shown at $-27 \mathrm{mV}$ is included for comparison but was not used in the calculations of the linear regression analysis because slight membrane rectification occurred at this depolarized potential. The extrapolation was obtained from membrane measurements in the ohmic portion of the current-voltage plot.

A

CONTROL TETANUS

B
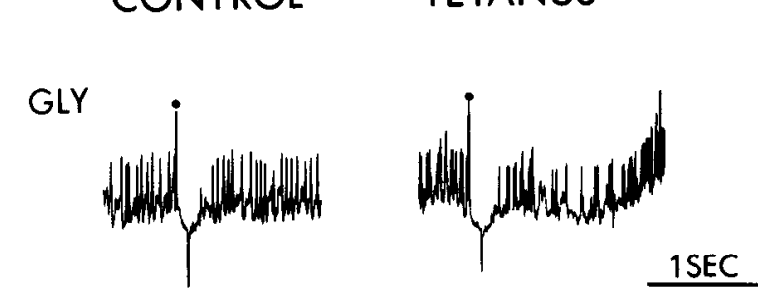

PRE

POST
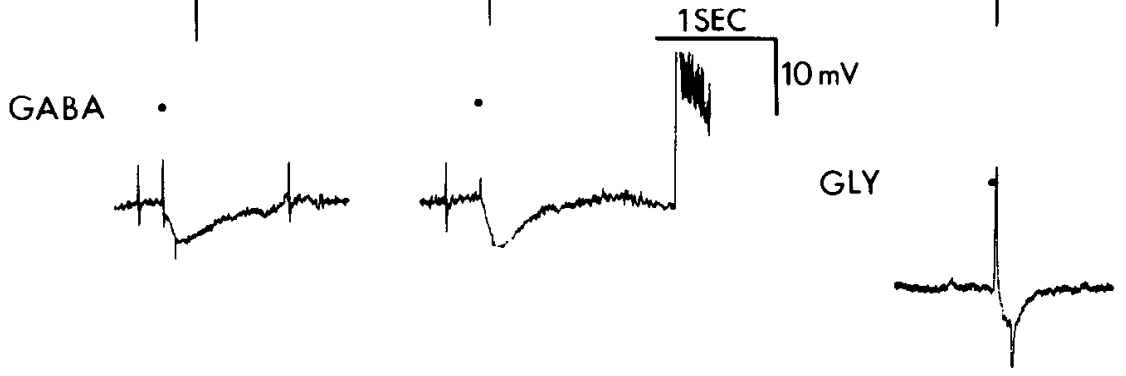

GLY

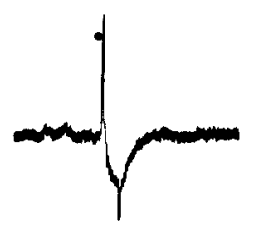

STRYCH

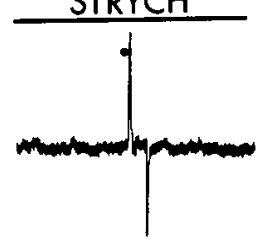

TETANUS

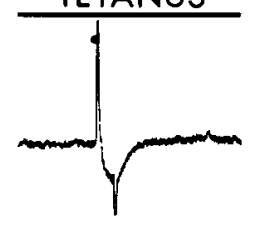

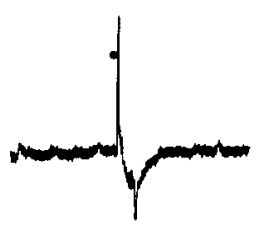

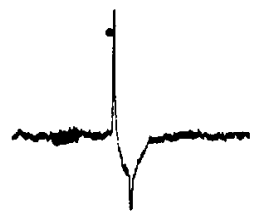

Figure 13. Tetanus toxin has no effect on postsynaptic responses to iontophoretically applied inhibitory amino acids glycine $(52 \mathrm{nA}, 100 \mathrm{msec})$ and GABA $(34 \mathrm{nA}, 100 \mathrm{msec})(A)$. Records shown in $A$ are before and $1 \mathrm{hr}$ after the addition of tetanus toxin $\left(10^{-3} \mathrm{gm} / \mathrm{ml}\right)$ when PDE were established. Pressure application strychnine $(0.1 \mathrm{~mm})$ rapidly and reversibly eliminated the response to iontophoretically applied glycine $(38 \mathrm{nA}, 100 \mathrm{msec})(B)$. Pressure application of tetanus toxin $\left(10^{-3} \mathrm{gm} / \mathrm{ml}\right) \mathrm{had} \mathrm{no}$ effect on the postsynaptic response to glycine. 
duced by tetanus toxin in spinal cord cultures are eliminated when synaptic transmission is blocked by high magnesium. Simultaneous intracellular recordings from a spinal cord cell pair in a given microscopic field always reveal identical PDE frequencies in both neurons regardless of whether mono- or polysynaptic connections between the two cells can be demonstrated. This local synchrony suggests that each neuronal network develops its own synaptically mediated tetanus-PDE. Direct intracellular stimulation cannot evoke PDE and there is no effect of membrane hyperpolarization on the tetanusPDE frequency. In contrast to the spinal cord neurons, DRG neurons in culture have not been demonstrated to have synaptic input (Ransom et al., 1977c). These DRG neurons do not develop PDE or bursting in the presence of tetanus toxin. The high percentage of spinal cord neurons developing tetanus-PDE parallels the high incidence of excitatory synaptic activity in this culture system. In preparations of cortex and hippocampus treated with the convulsant penicillin, the depolarizing events produced are thought to represent giant or summed excitatory events (Matsumoto et al., 1969; Dichter and Spencer, 1969; Johnston and Brown, 1981).

Many tetanus-PDE appear to be triggered by excitatory synaptic events. Although elimination of all synaptic inhibition is not an absolute requirement for the production of tetanus-PDE, the convulsant action of tetanus toxin is associated with a concomitant reduction in observed inhibitory synaptic potentials which were found to be reduced in incidence and amplitude. This reduction in inhibition can produce increased polysynaptic excitation. In the monosynaptically connected excitatory pairs studied to date, no enhancement of the monosynaptic EPSP has been observed with tetanus (G. K. Bergey and $\mathrm{H}$. Bigalke, unpublished data). The extrapolated reversal potential of the tetanus-PDE, within the limitations of a single electrode technique, is in the range of the reversal potential determined for spinal excitatory connections. The reversal potential of the tetanus-PDE cannot be determined exactly, even with expanded tracings, because the plateau of the depolarizing shift is a complex of multiple action potentials with AHP. The average reversal potential for spinal cord neuron EPSPs using two electrodes is $-4 \pm 12 \mathrm{mV}$ (SD; Macdonald et al., 1983). This positive reversal potential for the tetanusPDE can represent either contributing excitatory synaptic potentials or intrinsic voltage-dependent ionic conductances.

Mouse spinal cord neurons in culture have a voltagedependent calcium action potential (Heyer et al., 1981a). Tetrodotoxin completely abolishes the tetanus-PDE in these cultures; no underlying calcium depolarizations are seen. Nevertheless, calcium conductances could be augmented by tetanus toxin. The convulsant bicuculline has been shown to augment these calcium components of the action potential (Heyer et al., 1981b). Other intrinsic mechanisms such as dendritic spikes, shown to be important in the hippocampus (Wong and Prince, 1979), could also contribute to increased excitation.

Whether the tetanus-PDE with abrupt depolarizations are distinct from those with identifiable preceding synaptic potentials is not known. The recent report (Jeffreys and Haas, 1982) that synchronous bursting in hippocampal neurons can occur in the absence of synaptic transmission indicates that other as yet undetermined nonsynaptic mechanisms can be important in network synchrony.

In a similar spinal cord culture system a report of postsynaptic alterations in passive and active membrane properties by tetanus toxin has been reported (Dimpfel, 1979). In this instance, however, the concentrations of toxin used were sufficient to produce synaptic blockade at the time of assay, and control resting membrane potentials were quite low (average, $-33 \mathrm{mV}$ ) for this preparation; therefore, these results are difficult to interpret. We have not yet identified any alterations in passive or active membrane parameters produced by tetanus toxin during the development of tetanus-PDE during continuous recordings (G. K. Bergey and $\mathrm{H}$. Bigalke, unpublished data). No changes in membrane potential have been seen in intact preparations (Wiegand and Wellhöner, 1979). In a synaptosomal preparation using the lipophilic cation tetraphenylphosphonium, shifts in membrane potential with tetanus have been measured (Ramos et al., 1979). Long-term tetanus toxin exposure could lead to denervation changes in the postsynaptic cell population, but such changes are unlikely to be important in the production of the acute convulsant phenomena observed.

The evidence that tetanus affects inhibitory pathways in intact spinal cord (Brooks et al., 1957; Genisman et al., 1967) has suggested that tetanus may preferentially affect glycinergic transmission, since glycine is a likely candidate for ventral horn inhibition (see review by Duggan, 1978). There is evidence that axosomatic and proximal axodendritic terminals on the motoneurons may be glycinergic (Price et al., 1976); therefore, the apparent preference of tetanus for affecting glycinergic transmission could be explained on a morphological basis. Indeed, injection of tetanus toxin directly into the neuropil has shown tetanus to affect additional inhibitory connections (e.g., presynaptic inhibition) where GABA is the likely transmitter (Sverdlov and Alekseeva, 1966; Curtis et al., 1973; Davies and Tongroach, 1979). Therefore, the preference of action of tetanus toxin for a specific inhibitory neurotransmitter system is unresolved.

The neurotransmitters mediating inhibition in these spinal cord cultures remain to be definitely established, but data, including studies of convulsant action, suggest both glycinergic and GABAergic inhibition are present (Ransom et al., 1977a; Barker and Ransom, 1978; Macdonald and Barker, 1978; Nelson et al., 1981). Recordings from discrete monosynaptic inhibitory cell pairs have demonstrated IPSPs with at least two distinct time courses (Nelson et al., 1981). Whether tetanus toxin preferentially reduces glycinergic transmission before GABAergic inhibition in these cultures, where there is equal access of toxin to all inhibitory synapses, remains to be determined. Some preliminary evidence (G. K. Bergey, unpublished results) suggests that the more persistent (i.e., more resistant) IPSPs during tetanus-PDE have relatively long half-times; these may be GABA mediated. Ultimately, all spontaneous and evoked syn- 
aptic transmission, both excitatory and inhibitory, is blocked by tetanus toxin in these cultures (Bergey et al., 1981). The spinal cord culture system particularly lends itself to the study of discrete monosynaptically connected cell pairs (Neale et al., 1978; Nelson et al., 1981). The relative effects of tetanus toxin on excitatory versus inhibitory transmission can be established by using such defined connections in this culture system.

\section{References}

Arena, J. M. (1974) Poisoning, p. 158, Charles C Thomas, Springfield, IL.

Barile, M. F., M. C. Hardegree, and M. Pittman (1970) Immunization against neonatal tetanus in New Guinea. III. The toxin neutralization test and the response of guinea pigs to tbe toxoids as used in the immunization schedules in New Guinea. Bull. WHO 43: 453-459.

Barker, J. L., and B. R. Ransom (1978) Amino acid pharmacology of mammalian central neurons grown in tissue culture. J. Physiol. (Lond.) 280: 331-354.

Bergey, G. K., P. G. Nelson, R. L. Macdonald, and W. H. Habig (1981) Tetanus toxin produces blockade of synaptic transmission in mouse spinal cord neurons in culture. Soc. Neu rosci. Abstr. 7: 439.

Bigalke, H., J. Ahnert-Hilger, and E. Habermann (1981a) Tetanus toxin and botulinum toxin inhibit acetylcholine release from but not calcium uptake into brain tissue. Naunyn Schmiedebergs Arch. Pharmacol. 316: 142-148.

Bigalke, H., I. Heller, B. Bizzini and E. Habermann (1981b) Tetanus toxin and botulinism $A$ toxin inhibit release and uptake of various transmitters as studied with particulate preparations from rat brain and spinal cord. Naunyn Schmiedebergs Arch. Pharmacol. 316: 244-251.

Bizzini, B., A. Turpin, and M. Raynaud (1973) On the structure of tetanus toxin. Naunyn Schmiedebergs Arch. Pharmacol. 276: 271-285.

Bottenstein, J. E., and G. H. Sato (1979) Growth of a rat neuroblastoma cell line in serum-free supplemented medium. Proc. Natl. Acad. Sci. U. S. A. 76: 514-517.

Brooks, V. B., D. R. Curtis, and J. C. Eccles (1957) The action of tetanus toxin on the inhibition of motoneurons. J. Physiol. (Lond.) 135: 655-672.

Curtis, D. R. (1959) Pharmacological investigations upon inhibition of spinal motoneurons. J. Physiol. (Lond.) 145: 175192.

Curtis, D. R., and W. C. DeGroat (1968) Tetanus toxin and spinal inhibition. Brain Res. 10: 208-212.

Curtis, D. R., L. Hösli, and G. A. R. Johnston (1968) A pharmacological study of the depression of spinal neurons by glycine and related amino acids. Exp. Brain Res. 6: 1-18.

Curtis, D. R., A. W. Duggan, and G. A. R. Johnston (1971) The specificity of strychnine as a glycine antagonist in the mammalian spinal cord. Exp. Brain Res. 12: 547-565.

Curtis, D. R., D. Felix, C. J. A. Game, and R. M. McCulloch (1973) Tetanus toxin and the synaptic release of GABA. Brain Res. 51: 358-362.

Davidoff, R. A., M. H. Aprison, and R. Werman (1969) The effects of strychnine on the inhibition of interneurons by glycine and $\gamma$-amino butyric acid. Int. J. Neuropharmacol. 8 : 191-194.

Davies, J., and P. Tongroach (1979) Tetanus toxin and synaptic inhibition in the substantia nigra and striatum of the rat. J. Physiol. (Lond.) 290: 23-36.

del Castillo, J., and B. Katz (1954) The effect of magnesium on the activity of motor nerve endings. J. Physiol. (Lond.) 124: 553-559.

Dichter, M. A., and W. A. Spencer (1969) Penicillin-induced interictal discharges from the cat hippocampus. I. Characteristics and topographical features. J. Neurophysiol. 32: 649662.

Dimpfel, W. (1979) Hyperexcitability of cultured central nervous system neurons caused by tetanus toxin. Exp. Neurol. 65: 53-65.

Dimpfel, W., and E. Habermann (1977) Binding characteristics of ${ }^{125}$ I-labelled tetanus toxin to primary tissue cultures from mouse embryonic CNS. J. Neurochem. 29: 1111-1120.

Dimpfel, W., R. T. C. Huang, and E. Habermann (1977) Gangliosides in nervous tissue cultures and binding of ${ }^{125} \mathrm{I}-\mathrm{la}$ belled tetanus toxin, a neuronal marker. J. Neurochem. 29: 329-334.

Duggan, A. W. (1978) Pharmacology of mammalian central inhibition. Int. Rev. Physiol. 17: 119-148.

Erdmann, G., H. Wiegand, and H. H. Wellhöner (1975) Intraaxonal and extraaxonal transport of ${ }^{125} I$ tetanus toxin in early local tetanus. Naunyn Schmiedebergs Arch. Pharmacol. 290: $357-373$.

Erdmann, G., A. Hanauski, and H. H. Wellhöner (1981) Intraspinal distribution and reaction in the grey matter with tetanus toxin of intracisternally injected anti-tetanus toxoid $\mathrm{F}\left(\mathrm{ab}^{\prime}\right)_{2}$ fragments. Brain Res. 211: 367-377.

Evans, M. H. (1972) Tetrodotoxin, saxitoxin and related substances: Their application in neurobiology. Int. Rev. Neurobiol. 15: 83-166.

Genisman, Y. Y., M. V. D'Yakonova, and G. N. Kryzhanorsky (1967) Effect of tetanus toxin on morphological and functional site of synapses in spinal motoneurons. Bull. Exp. Biol. Med. 64: 1203-1206.

Giller, E. L., B. K. Schrier, A. Schainberg, H. R. Fisk, and P. G. Nelson (1973) Increased choline acetyltransferase activity in combined cultures of spinal cord and muscle cells from the mouse. Science 182: 588-589.

Gushchin, I. S., S. N. Koznechkin, and Y. S. Sverdlov (1969) On the presynaptic nature of the suppression of postsynaptic inhibition by tetanus toxin. Dokl. Akad. Nauk. SSSR 187: $685-688$.

Habermann, E. (1978) Tetanus. In Handbook of Clinical Neurology, P. J. Vinken and G. W. Bruyn, eds., Vol. 33, pp. 491547, Elsevier-North Holland Publishing Co., Amsterdam.

Habermann, E., and W. Dimpfel (1973) Distribution of ${ }^{125} \mathrm{I}$ tetanus toxin and ${ }^{125} \mathrm{I}$ toxoid in rats with generalized tetanus, as influenced by antitoxin. Naunyn Schmiedebergs Arch. Pharmacol. 276: 327-340.

Habermann, E., F. Dreyer, and H. Bigalke (1980) Tetanus toxin blocks neuromuscular transmission in vitro like Botulinum A toxin. Naunyn Schmiedebergs Arch. Pharmacol. 311: 33-40.

Heyer, E. J., R. L. Macdonald, G. K. Bergey, and P. G. Nelson (1981a) Calcium-dependent action potentials in mouse spinal cord neurons in cell culture. Brain Res. 200: 408-415.

Heyer, E. J., L. M. Nowak, and R. L. Macdonald (1981b) Bicuculline: A convulsant with synaptic and nonsynaptic actions. Neurology (NY) 31: 1381-1390.

Jeffreys, J. G. R., and H. L. Haas (1982) Synchronized bursting of CA 1 hippocampal pyramidal cells in the absence of synaptic transmission. Nature 300: 448-450.

Johnston, D., and T. H. Brown (1981) Giant synaptic potential hypothesis for epileptiform activity. Science 211: 294-297.

Katz, B., and R. Miledi (1963) A study of spontaneous miniature potentials in spinal motoneurons. J. Physiol. (Lond.) 168: 389-422.

Ledley, F. D., G. Lee, L. D. Kohn, W. H. Habig, and M. C. Hardegree (1977) Tetanus toxin interactions with thyroid plasma membranes. J. Biol. Chem. 252: 4049-4055.

Macdonald, R. L., and J. L. Barker (1978) Specific antagonism of GABA-mediated postsynaptic inhibition in cultured spinal 
cord neurons: A common mode of convulsant action. Neurology (NY) 28: 325-330.

Macdonald, R. L., and J. L. Barker (1981) Neuropharmacology of spinal cord neurons in primary dissociated cell culture. In Excitable Cells in Tissue Culture, P. G. Nelson and M. Lieberman, eds., pp. 81-110, Plenum Press, New York.

Macdonald, R. L., G. K. Bergey, and W. H. Habig (1979) Convulsant action of tetanus toxin on mammalian neurons in cell culture. Neurology (Minneap.) 29: 585.

Macdonald, R. L., R. Y. K. Pun, E. A. Neale, and P. G. Nelson (1983) Synaptic interactions between mammalian central neurons in cell culture. I. The reversal potential for excitatory postsynaptic potentials. J. Neurophysiol. 6: 1428-1441.

Matsumoto, H., G. F. Ayala, and R. J. Gumnit (1969) Neuronal behavior and triggering mechanism in cortical epileptic focus. J. Neurophysiol. 32: 688-703.

Mellanby, J., and J. Green (1981) How does tetanus toxin act? Neuroscience 6: 281-300.

Mellanby, J., and P. A. Thompson (1972) The effect of tetanus toxin at the neuromuscular junction of the goldfish. J. Physiol. (Lond.) 224: 407-419.

Mirsky, R., L. M. B. Wendon, P. Black, C. Stolsin, and D. Bray (1978) Tetanus toxin, a cell surface marker for neurons in culture. Brain Res. 148:251-259.

Neale, E. A., R. L. Macdonald, and P. G. Nelson (1978) Intracellular horseradish peroxidase injection for correlation of light and electron microscopic anatomy with synaptic physiology of cultured mouse spinal cord neurons. Brain Res. 152: 265-282.

Nelson, P. G., E. A. Neale, and R. L. Macdonald (1981) Electrophysiological and structural studies of neurons in dissociated cell cultures of the central nervous system. In Excitable Cells in Tissue Culture, P. G. Nelson and M. Lieberman, eds., pp. 39-80, Plenum Press, New York.

Pastan, I., M. Willingham, W. Anderson, and M. Gallo (1977) Localization of serum-derived $\alpha_{2}$ macroglobulin in cultured cells and decrease after Maloncy sarcoma virus transformation. Cell 12: 609-617.

Price, D. L., and J. W. Griffin (1981) Immunocytochemical localization of tetanus toxin to synapses of spinal cord. Neurosci. Lett. 23: 149-155.

Price, D. L., J. W. Griffin, A. Young, K. Peck, and A. Stocks (1975) Tetanus toxin: Direct evidence for retrograde intraaxonal transport. Science 188: 945-947.

Price, D. L., A. Stocks, J. W. Griffin, A. Young, and K. Peck (1976) Glycine-specific synapses in rat spinal cord: Identification by electron microscope autoradiography. J. Cell Biol. 68: 389-395.

Price, D. L., J. W. Griffin, and K. Peck (1977) Tetanus toxin: Evidence for binding at presynaptic nerve endings. Brain Res. 121: 379-384.
Price, D. L., P. Carroll, J. W. Griffin, and J. Morris (1978) Tetanus toxin: Immunocytochemical evidence for retrograde axonal transport. J. Neuropathol. Exp. Neurol. 37: 674.

Ramos, S., E. P. Grollman, P. Lazo, S. A. Dyer, W. H. Habig, M. C. Hardegree, H. R. Kaback, and L. D. Kohn (1979) Effect of tetanus toxin on the accumulation of the permeant lyophilic cation tetraphenylphosphonium by guinea pig brain synaptosomes. Proc. Natl. Acad. Sci. U. S. A. 76: 4783-4787.

Ransom, B. R., P. N. Bullock, and P. G. Nelson (1977a) Mouse spinal cord in cell culture. III. Neuronal chemosensitivity and its relationship to synaptic activity. J. Neurophysiol. 40 : 1163-1177.

Ransom, B. R., C. N. Christian, P. N. Bullock and P. G. Nelson (1977b) Mouse spinal cord in cell culture. II. Synaptic activity and circuit behavior. J. Neurophysiol. 40: 1151-1162.

Ransom, B. R., E. Neale, M. Henkart, P. N. Bullock, and P. G. Nelson (1977c) Mouse spinal cord in cell culture. I. Morphology and intrinsic neuronal electrophysiologic properties. J. Neurophysiol. 40: 1132-1150.

Schnaar, R. L., and A. E. Schaffner (1981) Separation of cell types from embryonic chicken and rat spinal cord: Characterization of motoneuron-enriched fractions. J. Neurosci. 1: 204-217.

Schwab, M. E., and H. Thoenen (1976) Electron microscopic evidence for trans-synaptic migration of tetanus toxin in spinal cord motoneurons: An autoradiographic and morphometric study. Brain Res. 105: 213-227.

Schwab, M. E., K. Suda, and H. Thoenen (1979) Selective retrograde trans-synaptic transfer of a protein, tetanus toxin, subsequent to its retrograde axonal transport. J. Cell Biol. 82: 798-810.

Spector, W. S. (1956) Handbook of Toxicology, p. 286, W. B. Saunders Co., Philadelphia.

Sverdlov, Y. S., and V. I. Alekseeva (1966) Effects of tetanus toxin on presynaptic inhibition in the spinal cord. Fed. Proc. (Transl. Suppl.) 25: T931-T935.

Van Heyningen, W. E. (1974) Gangliosides as membrane receptors for tetanus toxin, cholera toxin and serotonin. Nature 207: 642-643.

Wiegand, H., and H. H. Wellhöner (1979) Electrical excitability of motoneurons in early local tetanus. Naunyn-Schmiedebergs Arch. Pharmacol. 308: 71-76.

Wong, R. K. S., and D. A. Prince (1979) Dendritic mechanisms underlying penicillin-induced epileptiform activity. Science 204: 1228-1231.

Yavin, E., Z. Yavin, W. H. Habig, M. C. Hardigree, and L. D. Kohn (1981) Tetanus toxin association with developing neuronal cell cultures: Kinetic parameters and evidence for ganglioside-mediated internalization. J. Biol. Chem. 256: 70147022 . 